\title{
Coercivity Properties for Sequences of Lower Semicontinuous Functions on Metric Spaces
}

\author{
D. Motreanu' ${ }^{1}$ and V. V. Motreanu ${ }^{2}$ \\ ${ }^{1}$ Département de Mathématiques, Université de Perpignan Via Domitia, 66860 Perpignan, France \\ ${ }^{2}$ Department of Mathematics, Ben Gurion University of the Negev, 84105 Be'er Sheva, Israel \\ Correspondence should be addressed to V. V. Motreanu; motreanu@post.bgu.ac.il
}

Received 28 August 2013; Accepted 7 October 2013

Academic Editor: Salvador Hernandez

Copyright (C) 2013 D. Motreanu and V. V. Motreanu. This is an open access article distributed under the Creative Commons Attribution License, which permits unrestricted use, distribution, and reproduction in any medium, provided the original work is properly cited.

The paper presents various results studying the asymptotic behavior of a sequence of lower semicontinuous functions on a metric space. In particular, different coercivity properties are obtained extending and refining previous results. The specific features and the structure of the terms of the sequence are used to construct appropriate quantities relevant in the verification of Palais-Smale compactness type conditions.

\section{Introduction and Main Results}

Let $X$ be a complete metric space endowed with the metric $d$. We recall from De Giorgi et al. [1] the notion of strong slope of a lower semicontinuous function $f: X \rightarrow \mathbb{R} \cup\{+\infty\}$ (which is not identically $+\infty)$ at a point $u \in \operatorname{dom}(f):=\{w \in X$ : $f(w)<+\infty\}$ :

$$
|\nabla f|(u):= \begin{cases}0, & \text { if } u \text { is a local } \\ \text { minimizer of } f, \\ \limsup _{v \rightarrow u} \frac{f(u)-f(v)}{d(u, v)}, & \text { otherwise. }\end{cases}
$$

If $X$ is a Banach space and $f \in C^{1}(X, \mathbb{R})$, then $|\nabla f|(u)=$ $\left\|f^{\prime}(u)\right\|$ for all $u \in X$.

Let $F: X \rightarrow \mathbb{R}$ be a function satisfying the property.

$\mathrm{H}(F)$ : there exist constants $\gamma_{1}, \gamma_{2}>0$ such that

$$
d(u, v)<\gamma_{1} \Longrightarrow|F(u)-F(v)|<\gamma_{2} \quad(\text { for } u, v \in X)
$$

Let $f_{n}: X \rightarrow \mathbb{R} \cup\{+\infty\}(n \in \mathbb{N})$ be a sequence of lower semicontinuous functions. This paper develops a general approach for studying the asymptotic behavior of this sequence with respect to $F$. An aspect which makes our approach general and natural is that we do not require the sequence $\left\{f_{n}\right\}$ to admit a limit (in any sense); see also Remarks 7, 9, and 11 below. We introduce the notation

$$
\alpha^{F}\left(\left\{f_{n}\right\}\right):=\lim _{r \rightarrow+\infty} \lim _{n \rightarrow \infty} \inf _{\frac{\inf }{[F>r]}} f_{n} .
$$

Here and throughout the paper, for all $r>0$ we denote

$$
[F>r]:=\{w \in X: F(w)>r\},
$$

while $\overline{[F>r]}$ and $\operatorname{int}[F>r]$ stand for the closure and the interior of $[F>r]$, respectively. The expression $\alpha^{F}\left(\left\{f_{n}\right\}\right)$ always exists, generally belonging to $\mathbb{R} \cup\{ \pm \infty\}$. Equivalent expressions to $\alpha^{F}\left(\left\{f_{n}\right\}\right)$ can be given, for instance replacing $\overline{[F>r]}$ by $[F>r]$ or by $[F \geq r]:=\{w \in X: F(w) \geq r\}$ (see Lemma 16 below).

In what follows, we will always assume that.

$\mathrm{H}^{F}\left(\left\{f_{n}\right\}\right)$ : there holds $\alpha^{F}\left(\left\{f_{n}\right\}\right)>-\infty$.

For instance, $\mathrm{H}^{F}\left(\left\{f_{n}\right\}\right)$ is satisfied if $\left\{f_{n}\right\}$ is uniformly bounded below.

In the following we state our main result, which studies the asymptotic behavior of a sequence of lower semicontinuous functions.

Theorem 1. Let $X$ be a complete metric space, let $F: X \rightarrow \mathbb{R}$ be a function satisfying $\mathrm{H}(F)$, and let $f_{n}: X \rightarrow \mathbb{R} \cup\{+\infty\}$ 
( $n \in \mathbb{N}$ ) be a sequence of lower semicontinuous functions satisfying $\alpha^{F}\left(\left\{f_{n}\right\}\right) \in \mathbb{R}$. Then, for every $\varepsilon>0$, there exist a subsequence $\left\{f_{n_{k}}\right\}$ (depending on $\varepsilon$ ) of $\left\{f_{n}\right\}$ and a number $k_{\varepsilon} \in \mathbb{N}$ such that for each $k \geq k_{\varepsilon}$ one finds $u_{k, \varepsilon} \in \operatorname{dom}\left(f_{n_{k}}\right)$ satisfying

$$
\begin{gathered}
\alpha^{F}\left(\left\{f_{n}\right\}\right)-\varepsilon^{2}<f_{n_{k}}\left(u_{k, \varepsilon}\right)<\alpha^{F}\left(\left\{f_{n}\right\}\right)+\varepsilon^{2}, \\
\left|\nabla f_{n_{k}}\right|\left(u_{k, \varepsilon}\right) \leq \varepsilon, \\
F\left(u_{k, \varepsilon}\right)>\frac{1}{\varepsilon} .
\end{gathered}
$$

In particular, there are a subsequence $\left\{f_{m_{\ell}}\right\}$ of $\left\{f_{n}\right\}$ and elements $u_{\ell} \in \operatorname{dom}\left(f_{m_{\ell}}\right)$ such that

$$
\begin{gathered}
f_{m_{\ell}}\left(u_{\ell}\right) \longrightarrow \alpha^{F}\left(\left\{f_{n}\right\}\right), \quad\left|\nabla f_{m_{\ell}}\right|\left(u_{\ell}\right) \longrightarrow 0, \\
F\left(u_{\ell}\right) \longrightarrow+\infty \quad \text { as } \ell \longrightarrow \infty .
\end{gathered}
$$

The proof of Theorem 1 is done in Section 2.

Note that, due to the hypothesis that $\alpha^{F}\left(\left\{f_{n}\right\}\right) \in \mathbb{R}$, at least a subsequence of functions $f_{n}$ is not identically $+\infty$ and the sets $[F>r]$ are nonempty for all $r \in \mathbb{R}$.

We say that a sequence $\left\{u_{n}\right\} \subset X$ is F-bounded if the sequence $\left\{F\left(u_{n}\right)\right\} \subset X$ is bounded. We introduce the following notions of Palais-Smale condition and coercivity relative to the function $F$.

Definition 2. Let $f_{n}: X \rightarrow \mathbb{R} \cup\{+\infty\}(n \in \mathbb{N})$ be lower semicontinuous functions which are not identically $+\infty$. We say that the sequence $\left\{f_{n}\right\}$ satisfies the Palais-Smale condition relative to $F$ (condition (PS) ${ }^{F}$, for short) if whenever $\left\{f_{n_{k}}\right\}$ is a subsequence of $\left\{f_{n}\right\}$ and $\left\{u_{k}\right\} \subset X$ is a sequence such that $\left\{f_{n_{k}}\left(u_{k}\right)\right\}$ is bounded and $\left|\nabla f_{n_{k}}\right|\left(u_{k}\right) \rightarrow 0$ as $k \rightarrow \infty$, then $\left\{u_{k}\right\}$ is F-bounded.

Definition 3. Assume that the function $F$ satisfies in addition the requirement that $\sup _{X} F=+\infty$. We say that the sequence $f_{n}: X \rightarrow \mathbb{R} \cup\{+\infty\}(n \in \mathbb{N})$ is F-coercive if $\alpha^{F}\left(\left\{f_{n}\right\}\right)=+\infty$.

If $(X,\|\cdot\|)$ is a Banach space, $F=\|\cdot\|$, and $f_{n} \equiv f$ for all $n \in \mathbb{N}$, then we retrieve the usual notion of coercivity.

We state the following result on the F-coercivity of a sequence of lower semicontinuous functions.

Corollary 4. Let $X$ be a complete metric space, let $F: X \rightarrow \mathbb{R}$ be a function satisfying $\mathrm{H}(F)$ and $\sup _{X} F=+\infty$, and let $f_{n}$ : $X \rightarrow \mathbb{R} \cup\{+\infty\}(n \in \mathbb{N})$ be a sequence of lower semicontinuous functions satisfying $\mathrm{H}^{F}\left(\left\{f_{n}\right\}\right)$. If $\left\{f_{n}\right\}$ satisfies condition (PS) $)^{F}$, then the sequence $\left\{f_{n}\right\}$ is $F$-coercive.

The proof of Corollary 4 is given in Section 2.

As an immediate consequence of Corollary 4, in a Banach space we have the following.

Corollary 5. Let $(X,\|\cdot\|)$ be a Banach space, and let $f_{n}: X \rightarrow$ $\mathbb{R} \cup\{+\infty\}(n \in \mathbb{N})$ be a sequence of lower semicontinuous functions satisfying $\mathrm{H}^{\|\cdot\|}\left(\left\{f_{n}\right\}\right)$. If $\left\{f_{n}\right\}$ satisfies condition (PS) $)^{\|\cdot\|}$, then the sequence $\left\{f_{n}\right\}$ is $\|\cdot\|$-coercive.
Consider now the particular case in Theorem 1 when the number $\alpha^{F}\left(\left\{f_{n}\right\}\right)$ in $\mathrm{H}^{F}\left(\left\{f_{n}\right\}\right)$ is also a lim inf of a given lower semicontinuous function $f: X \rightarrow \mathbb{R} \cup\{+\infty\}$. Setting

$$
\alpha^{F}(f):=\liminf _{F(v) \rightarrow+\infty} f(v) \stackrel{\text { def }}{=} \lim _{r \rightarrow+\infty} \frac{\inf f}{[F>r]}
$$

the hypothesis $\alpha^{F}\left(\left\{f_{n}\right\}\right) \in \mathbb{R}$ in Theorem 1 is obviously satisfied if we assume the conditions

$$
\alpha^{F}\left(\left\{f_{n}\right\}\right)=\alpha^{F}(f) ; \quad \alpha^{F}(f) \in \mathbb{R} .
$$

It will be noted in Lemma 19 that the first condition in (10) is satisfied if the following is assumed. one has

$\mathrm{H}^{F}\left(\left\{f_{n}\right\}, f\right)$ : there exists $r_{0} \in \mathbb{R}$ such that for every $r \geq r_{0}$

(i) for every $u \in \operatorname{dom}(f) \cap \overline{[F>r]}$, there exists a sequence $\left\{u_{n}\right\}$ such that $u_{n} \rightarrow u$ and $f_{n}\left(u_{n}\right) \rightarrow f(u)$ as $n \rightarrow$ $\infty$;

(ii) $\liminf _{n \rightarrow \infty} \inf _{\overline{[F>r]}} f_{n} \geq \inf _{\overline{[F>r]}} f$.

Concerning the second condition in (10), we have the following simple characterization: given $c \in \mathbb{R}$, we have that $c=\alpha^{F}(f)$ if and only if,

$\forall a<c$, there exists $r \in \mathbb{R}$ such that $[f \leq a] \subset[F \leq r]$

$$
\forall a>c, \forall r \in \mathbb{R} \text {, we have }[f \leq a] \not \subset[F \leq r]
$$

(see Lemma 17 below). With the above comments, the following result is a consequence of Theorem 1 .

Corollary 6 (Corvellec $\left[2\right.$, Theorem $\left.\left.1^{\prime}\right]\right)$. Let $X$ be a complete metric space, let $F: X \rightarrow \mathbb{R}$ be a function bounded on bounded subsets of $X$ and satisfying $\mathrm{H}(F)$, and let $f, f_{n}: X \rightarrow$ $\mathbb{R} \cup\{+\infty\}(n \in \mathbb{N})$ be lower semicontinuous functions satisfying that,

for all $u \in \operatorname{dom}(f)$, there exists

$$
\begin{gathered}
u_{n} \longrightarrow u \text { with } f_{n}\left(u_{n}\right) \longrightarrow f(u), \\
\text { for all closed } Y \subset X, \liminf _{n \rightarrow \infty}\left(\inf _{Y} f_{n}\right) \geq \inf _{Y} f .
\end{gathered}
$$

Assume (11) for some $c \in \mathbb{R}$. Then there exist a subsequence $\left\{f_{n_{k}}\right\}$ of $\left\{f_{n}\right\}$ and a sequence $\left\{u_{k}\right\} \subset X$ such that $f_{n_{k}}\left(u_{k}\right) \rightarrow c$, $\left|\nabla f_{n_{k}}\right|\left(u_{k}\right) \rightarrow 0$, and $F\left(u_{k}\right) \rightarrow+\infty$ as $k \rightarrow \infty$.

Remark 7. The number $c \in \mathbb{R}$ in Corollary 6 is necessarily $c=\alpha^{F}(f)=\alpha^{F}\left(\left\{f_{n}\right\}\right)$. Moreover, hypotheses (12)-(13) are particular cases of $\mathrm{H}^{F}\left(\left\{f_{n}\right\}, f\right)$ (i)-(ii) that involve only sets of the form $Y=\overline{[F>r]}$. Hence the hypotheses of Corollary 6 , namely, (11), (12), (13), and $c \in \mathbb{R}$, imply that $\alpha^{F}\left(\left\{f_{n}\right\}\right) \in \mathbb{R}$. Therefore, [2, Theorem $1^{\prime}$ ] (i.e., Corollary 6) is retrieved as a consequence of Theorem 1 (and then the hypothesis that $F$ is bounded on bounded subsets of $X$ is not even needed). As seen from Example 12, Theorem 1 is actually more general 
than $\left[2\right.$, Theorem $\left.1^{\prime}\right]$, and besides it does not need an auxiliary function $f$ in its hypotheses.

Next, we note that the hypothesis $\mathrm{H}^{F}\left(\left\{f_{n}\right\}\right)$ in Corollary 4 is satisfied if we assume the conditions

$$
\alpha^{F}\left(\left\{f_{n}\right\}\right)=\alpha^{F}(f), \quad \alpha^{F}(f)>-\infty
$$

As noticed above, the first condition in (14) is satisfied if $\mathrm{H}^{F}\left(\left\{f_{n}\right\}, f\right)$ holds, which in turn is satisfied if (12) and (13) are assumed. The second condition in (14) is equivalent to the property $[F>b] \subset[f>a]$ for some $a, b \in \mathbb{R}$; that is,

$$
[f \leq a] \subset[F \leq b], \quad \text { for some } a, b \in \mathbb{R}
$$

(see Lemma 18). We thus have the following consequence of Corollary 4. Here, as in [2], it is said that $f$ satisfies condition (PSB) ${ }^{*}$ if whenever $\left\{f_{n_{k}}\right\}$ is a subsequence of $\left\{f_{n}\right\}$ and $\left\{u_{k}\right\} \subset X$ is a sequence such that $\left\{f_{n_{k}}\left(u_{k}\right)\right\}$ is bounded and $\left|\nabla f_{n_{k}}\right|\left(u_{k}\right) \rightarrow 0$ as $k \rightarrow \infty$, then $\left\{u_{k}\right\}$ is bounded.

Corollary 8 (Corvellec [2, Theorem 1]). Let X be a complete metric space, let $F: X \rightarrow \mathbb{R}$ be a function bounded on bounded subsets of $X$ satisfying $\mathrm{H}(F)$ and $\sup _{X} F=+\infty$, and let $f, f_{n}: X \rightarrow \mathbb{R} \cup\{+\infty\}(n \in \mathbb{N})$ be lower semicontinuous functions satisfying (12) and (13). If $f$ satisfies (15) and condition (PSB) ${ }^{*}$, then $f$ is F-coercive (i.e., $f(u) \rightarrow$ $+\infty$ as $F(u) \rightarrow+\infty)$.

Remark 9. From the above discussion, Corollary 8 (i.e., [2, Theorem 1]) is obtained as a consequence of Corollary 4 . In fact, Corollary 4 is more general (see Example 12) and does not rely on an auxiliary function $f$. In fact, on the one hand to study the coercivity of a function $f$ we do not need to look for a sequence $\left\{f_{n}\right\}$ as in Corollary 8 (in applications it seems to be more difficult to prove the existence of a sequence $\left\{f_{n}\right\}$ related to the function $f$ as in Corollary 8 than to prove the coercivity of $f$ itself). On the other hand, while studying the coercivity of a sequence $\left\{f_{n}\right\}$, the interest of Corollary 4 is to give sufficient conditions for the coercivity of the sequence $\left\{f_{n}\right\}$ without using an auxiliary function $f$. Finally, we note that in addition to the $F$-coercivity of $f$, the hypotheses of Corollary 8 imply also the $F$-coercivity of $\left\{f_{n}\right\}$, and so $\alpha^{F}\left(\left\{f_{n}\right\}\right)=\alpha^{F}(f)=+\infty$.

We also recall the following.

Corollary 10 (Corvellec [2, Corollary 1]). Let $(X,\|\cdot\|)$ be a Banach space, let $f, f_{n}: X \rightarrow \mathbb{R} \cup\{+\infty\}(n \in \mathbb{N})$ be bounded below, lower semicontinuous functions satisfying (12) and (13). Then $f$ is $\|\cdot\|$-coercive if and only if $f$ satisfies condition (PSB) ${ }^{*}$.

The sufficiency in Corollary 10 follows from Corollary 5 (or from Corollary 8). The necessity can be proved arguing by contradiction in the following way. If there are a subsequence $\left\{f_{n_{k}}\right\}$ of $\left\{f_{n}\right\}$ and a sequence $\left\{u_{k}\right\} \subset X$ such that $\left\{f_{n_{k}}\left(u_{k}\right)\right\}$ is bounded, $\left|\nabla f_{n_{k}}\right|\left(u_{k}\right) \rightarrow 0$ and $\left\|u_{k}\right\| \rightarrow+\infty$ as $k \rightarrow \infty$, then $f\left(u_{k}\right) \rightarrow+\infty$ as $k \rightarrow \infty$ (by the $\|\cdot\|$-coercivity of $f$ ).
Then, for every $n \in \mathbb{N}$, using (13) with the closed set $Y_{n}:=$ $\left\{u_{n}, u_{n+1}, \ldots\right\}$, we have

$$
\begin{aligned}
\liminf _{k \rightarrow \infty} f_{n_{k}}\left(u_{k}\right) & \geq \liminf _{k \rightarrow \infty}\left(\inf _{Y_{n}} f_{n_{k}}\right) \\
& \geq \liminf _{k \rightarrow \infty}\left(\inf _{Y_{n}} f_{k}\right) \geq \inf _{Y_{n}} f \longrightarrow+\infty
\end{aligned}
$$$$
\text { as } n \longrightarrow \infty \text {, }
$$

which contradicts the boundedness of the sequence $\left\{f_{n_{k}}\left(u_{k}\right)\right\}$.

Remark 11. Hypotheses (12) and (13) imply the first part of (10); that is, $\alpha^{\|\cdot\|}\left(\left\{f_{n}\right\}\right)=\alpha^{\|\cdot\|}(f)$. Hence, in view of Corollary 5, in place of assuming that $f$ and $f_{n}$ are bounded below in [2, Corollary 1] it would have been enough to assume that $\alpha^{\|\cdot\|}(f)>-\infty$, which in fact is implied just by the boundedness below of $f$. Corollary 5 is more general than Corollary 10 and its advantage is that it studies the coercivity of a sequence $\left\{f_{n}\right\}$ without dealing with an auxiliary function $f$. For the study of the coercivity of a function $f$ we do not need to involve a sequence of functions $\left\{f_{n}\right\}$ (see Corollary 31 below).

Example 12. (a) Let $f: \mathbb{R} \rightarrow \mathbb{R} \cup\{+\infty\}$ be a lower semicontinuous, even (i.e., $f(x)=f(-x)$ for all $x \in \mathbb{R}$ ) function which is not identically $+\infty$ on $\mathbb{R} \backslash\{0\}$, let the lower semicontinuous functions $f_{n}: \mathbb{R} \rightarrow \mathbb{R} \cup\{+\infty\}(n \in \mathbb{N})$ be given by

$$
f_{n}(x)= \begin{cases}f(x), & \text { if } x \geq 0, \\ +\infty, & \text { if } x<0,\end{cases}
$$

and let $F(x)=|x|$ for all $x \in \mathbb{R}$. Then we have $\alpha^{F}\left(\left\{f_{n}\right\}\right)=$ $\alpha^{F}(f)$. Condition $\mathrm{H}^{F}\left(\left\{f_{n}\right\}, f\right)$ (i) is not satisfied (thus, (12) is not satisfied) since, if $x \in \operatorname{dom}(f), x<0$, then for every sequence $x_{n} \rightarrow x$ we have $f_{n}\left(x_{n}\right)=+\infty>f(x)$ for $n \in \mathbb{N}$ large enough. So Corollaries 6, 8, and 10 cannot be applied, while Theorem 1 can be applied whenever $\alpha^{F}(f) \epsilon$ $\mathbb{R}$. Corollaries 4 and 5 can also be applied.

(b) Let $f: \mathbb{R} \rightarrow \mathbb{R}$ be defined by $f(x)=-e^{-2 x^{2}}$ for all $x \in \mathbb{R}$, let $f_{n}: \mathbb{R} \rightarrow \mathbb{R}(n \in \mathbb{N})$ be defined by $f_{n}(x)=$ $-e^{-x^{2}}-(1 / n)$ for all $x \in \mathbb{R}$, and let $F(x)=|x|$ for all $x \in \mathbb{R}$. Then we have $\alpha^{F}\left(\left\{f_{n}\right\}\right)=\alpha^{F}(f)=0$. Condition $\mathrm{H}^{F}\left(\left\{f_{n}\right\}, f\right)$ (ii) is not satisfied (so neither (13)) since for every $r>0$ we have

$$
\liminf _{n \rightarrow \infty} \inf _{|x| \geq r} f_{n}(x)=-e^{-r^{2}}<-e^{-2 r^{2}}=\inf _{|x| \geq r} f(x) .
$$

Hence we may apply Theorem 1 to the sequence $\left\{f_{n}\right\}$, but not Corollary 6 with $\left\{f_{n}\right\}$ and the chosen $f$. Besides being more general, the advantage of Theorem 1 is to study the asymptotic behavior of a sequence $\left\{f_{n}\right\}$ without an auxiliary function $f$ (if it exists) as in Corollary 6.

(c) Let $f: \mathbb{R} \rightarrow \mathbb{R}$ be defined by $f(x)=e^{|x|}$ for all $x \in \mathbb{R}$, let $f_{n}: \mathbb{R} \rightarrow \mathbb{R}(n \in \mathbb{N})$ be defined by $f_{n}(x)=$ $\left(x^{2} / 2\right)-(1 / n)$ for all $x \in \mathbb{R}$, and let $F(x)=|x|$ for all $x \in \mathbb{R}$. 
Then $\alpha^{F}\left(\left\{f_{n}\right\}\right)=\alpha^{F}(f)=+\infty$. Condition $\mathrm{H}^{F}\left(\left\{f_{n}\right\}, f\right)$ (ii) is not satisfied (so neither (13)) since for every $r>0$ we have

$$
\liminf _{n \rightarrow \infty} \inf _{|x| \geq r} f_{n}(x)=\frac{r^{2}}{2}<e^{r}=\inf _{|x| \geq r} f(x) .
$$

Corollary 4 (or Corollary 5) can be applied to $\left\{f_{n}\right\}$ (note that $\left\{f_{n}\right\}$ satisfies condition $\left.(\mathrm{PS})^{F}\right)$, while Corollaries 8 and 10 cannot be applied to $\left\{f_{n}\right\}$ and $f$ (however, $f$ is coercive).

(d) Let $(X,\|\cdot\|)$ be a Banach space and $F: X \rightarrow \mathbb{R}$ be a continuous function satisfying $\mathrm{H}(F)$. Let $g: \mathbb{R} \rightarrow$ $\mathbb{R} \cup\{+\infty\}$ and $h: \mathbb{R} \rightarrow \mathbb{R}$ be nondecreasing, lower semicontinuous functions with the property that there exists $r_{0}>0$ such that $h(r)<g(r)$ for all $r \geq r_{0}$ and $\lim _{r \rightarrow+\infty} h(r)=$ $\lim _{r \rightarrow+\infty} g(r)>-\infty$. Define $f=g \circ F$ and let $f_{n}: \mathbb{R} \rightarrow \mathbb{R}$ $(n \in \mathbb{N})$ be given by

$$
f_{n}(x)= \begin{cases}h(F(x))+s_{n}, & \text { if } F(x) \leq n, \\ +\infty, & \text { if } F(x)>n,\end{cases}
$$

where $\left\{s_{n}\right\} \subset \mathbb{R}$. Suppose $\liminf _{n \rightarrow \infty} s_{n}=0$. Then $\alpha^{F}\left(\left\{f_{n}\right\}\right)=$ $\alpha^{F}(f)$, but condition $\mathrm{H}^{F}\left(\left\{f_{n}\right\}, f\right)$ (ii) is not satisfied (so neither (13)) since for every $r>r_{0}$ we have

$$
\liminf _{n \rightarrow \infty} \inf _{F(x) \geq r} f_{n}(x)=h(r)<g(r)=\inf _{F(x) \geq r} f(x) .
$$

So, neither Corollary 6 nor Corollary 8 can be applied. Theorem 1 can be applied whenever $\alpha^{F}\left(\left\{f_{n}\right\}\right)=\lim _{r \rightarrow+\infty} h(r)+$ $\liminf _{n \rightarrow \infty} s_{n} \in \mathbb{R}$ (e.g., we do not need that $\liminf _{n \rightarrow \infty} s_{n}=$ 0 , and then $\left.\alpha^{F}\left(\left\{f_{n}\right\}\right)=\alpha^{F}(f)+\liminf _{n \rightarrow \infty} s_{n}\right)$ and its application is not related to some auxiliary function $f$. For Corollary 4 , we must necessarily have $\alpha^{F}\left(\left\{f_{n}\right\}\right)=+\infty$.

Example 13. (a) As examples of functions $F: X \rightarrow \mathbb{R}$ satisfying $\mathrm{H}(F)$, we can consider any Lipschitz continuous function on a metric space $X$, or any uniformly continuous function. For example, if $X$ is a metric space endowed with the metric $d$, then the function

$$
F(u)=d(u, A), \quad \forall u \in X,
$$

for some nonempty subset $A$ of $X$, satisfies hypothesis $\mathrm{H}(F)$ with any $\gamma_{1}=\gamma_{2}$, and if $X \backslash A$ is unbounded, then $F$ satisfies also that $\sup _{X} F=+\infty$ (so both Theorem 1 and Corollary 4 can be applied in this case). In particular, in the case where $(X,\|\cdot\|)$ is a Banach space, the function $F=\|\cdot\|$ satisfies $\mathrm{H}(F)$ and that $\sup _{X} F=+\infty$.

(b) Note that if $A$ is a bounded subset of the metric space $X$, then the expression $\alpha^{d(\cdot, A)}\left(\left\{f_{n}\right\}\right)$ (i.e., $\alpha^{F}\left(\left\{f_{n}\right\}\right)$ for $F(\cdot)=$ $d(\cdot, A))$ and the notion of $d(\cdot, A)$-coercivity do not depend on the choice of the set $A$ (for this reason, we refer to $d$-coercivity in place of $d(\cdot, A)$-coercivity). If $A$ is unbounded, then it is not anymore the case: for example, if $X=\mathbb{R}^{2}, A=\{(x, y) \in$ $\left.\mathbb{R}^{2}: y=0\right\}$, and $f: \mathbb{R}^{2} \rightarrow \mathbb{R}$ given by $f(x, y)=|y|$, then $f$ is $d(\cdot, A)$-coercive, but it is not norm coercive (taking the Euclidean norm and denoting by $d$ the induced distance).

(c) As another example of $F$ (which is not even continuous), let $X=\mathbb{R}, F: \mathbb{R} \rightarrow \mathbb{R}$ given by

$$
F(x)= \begin{cases}0, & \text { if }|x|<1 \\ x, & \text { if }|x| \geq 1\end{cases}
$$

The function $F$ satisfies $\mathrm{H}(F)$ with $\gamma_{1}=1, \gamma_{2}=2$. Note that for every $r \in \mathbb{R}$, we have $F(r+1)>r$ (so the sets $[F>r]$ are nonempty).

Remark 14. (a) If $F: X \rightarrow \mathbb{R}$ and $\widetilde{F}: X \rightarrow \mathbb{R}$ are two functions satisfying $\mathrm{H}(F)$ and that $\sup _{X} F=\sup _{X} \widetilde{F}=+\infty$, and if $F-\widetilde{F}$ is bounded, then the $F$-coercivity and the $\widetilde{F}$ coercivity of a sequence $\left\{f_{n}\right\}$ as in Corollary 4 are equivalent.

(b) If $X$ is a metric space endowed with two metrics $d$ and $\tilde{d}$ which induce the same topology, then a sequence $\left\{f_{n}\right\}$ as in Corollary 4 may be $d$-coercive and non- $\widetilde{d}$-coercive.

(c) Let $X$ be a metric space endowed with the metric $d$, let $F: X \rightarrow \mathbb{R}$ be a function satisfying $\mathrm{H}(F)$ and $\sup _{X} F=+\infty$, and let us define a new metric:

$$
\tilde{d}(x, y)=d(x, y)+|F(x)-F(y)|
$$

The topology induced by $\widetilde{d}$ is finer than the topology induced by $d$ (if $F$ is Lipschitz continuous with respect to $d$, then they are equivalent). Applying Corollary 4 to the metric $\tilde{d}$, if $d$ is bounded, then the F-coercivity is equivalent to the $\widetilde{d}$-coercivity. In this case, Corollary 4 yields that if $\left\{f_{n}\right\}$ is a sequence of lower semicontinuous functions with respect to $d$ (thus with respect to $\widetilde{d}$ ) satisfying $\mathrm{H}^{F}\left(\left\{f_{n}\right\}\right)$ and such that condition (PS) ${ }^{F}$ holds with respect to the metric $\widetilde{d}$, then $\left\{f_{n}\right\}$ is $\tilde{d}$-coercive.

The rest of the paper is organized as follows.

Section 2 contains the proofs of the results stated in Section 1, based on the Ekeland variational principle. Our approach in showing Theorem 1 relies on the ideas in the proof of Motreanu-Motreanu [3, Theorem 3.1], which is a different approach from the one of Corvellec [2].

Section 3 contains further applications of Theorem 1 and Corollary 4 to special classes of sequences of lower semicontinuous functions. Section 3.1 is concerned with the coercivity of a sequence of lower semicontinuous functions fulfilling $\mathrm{H}^{F}\left(\left\{f_{n}\right\}\right)$ in the case where liminf in the definition of $\alpha^{F}\left(\left\{f_{n}\right\}\right)$ is actually a limit. Section 3.2 studies the coercivity of a sequence of lower semicontinuous functionals which can be written as a sum of a locally Lipschitz function and a convex, lower semicontinuous function which is not identically $+\infty$. Section 3.3 deals with the coercivity of a continuously differentiable functional $f$ on a Banach space under a Palais-Smale type condition relative to a sequence of Galerkin approximations of $f$. Section 3.4 deals with the case of a constant sequence of lower semicontinuous functions. Section 3.5 discusses what happens if in place of hypothesis $\mathrm{H}^{F}\left(\left\{f_{n}\right\}\right)$ we consider the case where the limits in $\alpha^{F}\left(\left\{f_{n}\right\}\right)$ are interchanged.

\section{Proofs of Theorem 1, Corollary 4, and Additional Lemmas}

A basic ingredient in proving our results is the following version of the Ekeland variational principle (see Ekeland [4]). 
Theorem 15. Let $(X, d)$ be a complete metric space and let $f$ : $X \rightarrow \mathbb{R} \cup\{+\infty\}$ be a lower semicontinuous function which is bounded below and not identically $+\infty$. Then for every $\varepsilon>0$ and every $v \in X$ there exists $u \in X$ such that

$$
\begin{array}{r}
f(u) \leq f(v)-\varepsilon d(u, v), \quad f(w)>f(u)-\varepsilon d(w, u), \\
\forall w \in X \backslash\{u\} .
\end{array}
$$

We need the following preliminary lemma.

Lemma 16. If $F: X \rightarrow \mathbb{R}$ satisfies $\mathrm{H}(F)$, then for every $r \in \mathbb{R}$ one has,
(a) $\overline{[F>r]} \subset\left[F>r-\gamma_{2}\right]$,
(b) $[F>r] \subset \operatorname{int}\left[F>r-\gamma_{2}\right]$.

Proof. (a) Let $u \in \overline{[F>r]}$, and let $\left\{u_{n}\right\} \subset[F>r]$ be a sequence such that $u_{n} \rightarrow u$ as $n \rightarrow \infty$. Since, for $n$ sufficiently large, $d\left(u_{n}, u\right)<\gamma_{1}$, by hypothesis $\mathrm{H}(F)$ we have that $F(u)>F\left(u_{n}\right)-\gamma_{2}>r-\gamma_{2}$.

(b) Let $u \in[F>r]$, and let $v \in X$ be such that $d(v, u)<\gamma_{1}$. Then hypothesis $\mathrm{H}(F)$ yields $F(v)>F(u)-\gamma_{2}>r-\gamma_{2}$, which completes the proof.

Proof of Theorem 1. We denote $\alpha:=\alpha^{F}\left(\left\{f_{n}\right\}\right) \in \mathbb{R}$. Fix $\varepsilon \in$ $\left(0, \gamma_{1} / 2\right)$. By the definition of $\alpha$, it follows that there exists $r_{\varepsilon}>$ 0 such that

$$
\alpha-\varepsilon^{2}<\liminf _{n \rightarrow \infty} \frac{\inf }{[F>r]} f_{n}<\alpha+\varepsilon^{2}, \quad \forall r \geq r_{\varepsilon} .
$$

This implies that for every $r \geq r_{\varepsilon}$ we find a number $n_{r, \varepsilon} \in \mathbb{N}$ such that

$$
\alpha-\varepsilon^{2}<\inf _{[F>r]} f_{n}, \quad \forall n \geq n_{r, \varepsilon}
$$

and a subsequence $\left\{f_{n_{k}}^{(r)}\right\}$ (depending on $r$ ) of $\left\{f_{n}\right\}$ and a number $k_{r, \varepsilon} \in \mathbb{N}$ such that

$$
\inf _{[F>r]} f_{n_{k}}^{(r)}<\alpha+\varepsilon^{2}, \quad \forall k \geq k_{r, \varepsilon} .
$$

Denote

$$
\bar{r}_{\varepsilon}=\max \left\{r_{\varepsilon}, \frac{1}{\varepsilon}+\gamma_{2}, 2 \gamma_{2}\right\} .
$$

In particular, from (27), for $r=\bar{r}_{\varepsilon}$, we find a number $n_{\bar{r}_{\varepsilon}, \varepsilon} \in \mathbb{N}$ such that

$$
\alpha-\varepsilon^{2}<\inf _{\left[F>\bar{r}_{\varepsilon}\right]} f_{n}, \quad \forall n \geq n_{\bar{r}_{\varepsilon}, \mathcal{\varepsilon}},
$$

while from (28), for $r=2 \bar{r}_{\varepsilon}$ we find a subsequence $\left\{f_{n_{k}}\right\}$ (which is the subsequence $\left\{f_{n_{k}}^{\left(2 \bar{r}_{\varepsilon}\right)}\right\}$ ) of $\left\{f_{n}\right\}$ and a number $k_{2 \bar{r}_{\varepsilon}, \varepsilon}$ such that

$$
\inf _{\left[F>2 \bar{r}_{\varepsilon}\right]} f_{n_{k}}<\alpha+\varepsilon^{2}, \quad \forall k \geq k_{2 \bar{r}_{\varepsilon}, \varepsilon}
$$

Fix $k \geq k_{\varepsilon}:=\max \left\{n_{\bar{r}_{\varepsilon}, \varepsilon}, k_{2 \bar{r}_{\varepsilon}, \varepsilon}\right\}$. Using (31) we find a point

$$
v_{k, \varepsilon} \in \overline{\left[F>2 \bar{r}_{\varepsilon}\right]}
$$

for which one has

$$
f_{n_{k}}\left(v_{k, \varepsilon}\right)<\alpha+\varepsilon^{2} .
$$

Corresponding to the set $\overline{\left[F>\bar{r}_{\varepsilon}\right]}$ (see (29)), consider the function $\widetilde{f}_{n_{k}}: \overline{\left[F>\bar{r}_{\varepsilon}\right]} \rightarrow \mathbb{R} \cup\{+\infty\}$ as follows:

$$
\tilde{f}_{n_{k}}=\left.f_{n_{k}}\right|_{\left[\overline{\left[F>\bar{r}_{\varepsilon}\right.}\right]} .
$$

It is clear that the set $\overline{\left[F>\bar{r}_{\varepsilon}\right]}$ is a complete metric space with respect to the metric induced by $d$ and that the function $\widetilde{f}_{n_{k}}$ is lower semicontinuous. In addition, $\widetilde{f}_{n_{k}}$ is not identically $+\infty$ since by (32), (33), and (34) we have that $v_{k, \varepsilon} \in \operatorname{dom}\left(\tilde{f}_{n_{k}}\right)$. By (34), (30) and using that $n_{k} \geq k \geq k_{\varepsilon} \geq n_{\bar{r}_{\varepsilon}, \varepsilon}$, we see that

$$
\tilde{f}_{n_{k}}(w)=f_{n_{k}}(w)>\alpha-\varepsilon^{2}, \quad \forall w \in \overline{\left[F>\bar{r}_{\varepsilon}\right]} ;
$$

hence $\tilde{f}_{n_{k}}$ is bounded from below. Therefore we are in a position to apply Theorem 15 to the function $\tilde{f}_{n_{k}}$ on the complete metric space $\overline{\left[F>\bar{r}_{\varepsilon}\right]}$. Then there exists

$$
u_{k, \varepsilon} \in \overline{\left[F>\bar{r}_{\varepsilon}\right]}
$$

such that

$$
f_{n_{k}}\left(u_{k, \varepsilon}\right) \leq f_{n_{k}}\left(v_{k, \varepsilon}\right)-\varepsilon d\left(u_{k, \varepsilon}, v_{k, \varepsilon}\right),
$$

$f_{n_{k}}(w)>f_{n_{k}}\left(u_{k, \varepsilon}\right)-\varepsilon d\left(w, u_{k, \varepsilon}\right), \quad \forall w \in \overline{\left[F>\bar{r}_{\varepsilon}\right]} \backslash\left\{u_{k, \varepsilon}\right\}$.

In view of (33), we see that $f_{n_{k}}\left(v_{k, \varepsilon}\right)<+\infty$, which combined with (37) ensures that $u_{k, \varepsilon} \in \operatorname{dom}\left(f_{n_{k}}\right)$.

By (36) and Lemma 16 (a) we have that $u_{k, \varepsilon} \in \overline{\left[F>\bar{r}_{\varepsilon}\right]} \subset$ $\left[F>\bar{r}_{\varepsilon}-\gamma_{2}\right]$, which, in view of (29), yields $F\left(u_{k, \varepsilon}\right)>\bar{r}_{\varepsilon}-\gamma_{2} \geq$ $1 / \varepsilon$, so $(7)$ holds true.

Using (35), (36), (37), and (33) we have

$$
\begin{aligned}
\alpha-\varepsilon^{2}<f_{n_{k}}\left(u_{k, \varepsilon}\right) & \leq f_{n_{k}}\left(v_{k, \varepsilon}\right)-\varepsilon d\left(u_{k, \varepsilon}, v_{k, \varepsilon}\right) \\
& <\alpha+\varepsilon^{2}-\varepsilon d\left(u_{k, \varepsilon}, v_{k, \varepsilon}\right),
\end{aligned}
$$

which proves (5).

Let us show that

$$
u_{k, \varepsilon} \in \operatorname{int} \overline{\left[F>\bar{r}_{\varepsilon}\right]} .
$$

Let $\mu_{\varepsilon} \in\left(0, \gamma_{1}-2 \varepsilon\right)$. To get (40) it suffices to prove that

$$
d\left(w, u_{k, \varepsilon}\right)<\mu_{\varepsilon} \Longrightarrow w \in\left[F>\bar{r}_{\varepsilon}\right] .
$$

To see this, let $w$ satisfy $d\left(w, u_{k, \varepsilon}\right)<\mu_{\varepsilon}$. By (39), we have that $d\left(u_{k, \varepsilon}, v_{k, \varepsilon}\right)<2 \varepsilon$, which implies that $d\left(w, v_{k, \varepsilon}\right) \leq d\left(w, u_{k, \varepsilon}\right)+$ $d\left(u_{k, \varepsilon}, v_{k, \varepsilon}\right)<\mu_{\varepsilon}+2 \varepsilon<\gamma_{1}$. In view of $\mathrm{H}(F)$, this leads to $\left|F(w)-F\left(v_{k, \varepsilon}\right)\right|<\gamma_{2}$. On the other hand, by (32) and 
Lemma 16 (a), we know that $v_{k, \varepsilon} \in \overline{\left[F>2 \bar{r}_{\varepsilon}\right]} \subset\left[F>2 \bar{r}_{\varepsilon}-\gamma_{2}\right]$. It follows that $F(w)>F\left(v_{k, \varepsilon}\right)-\gamma_{2}>2 \bar{r}_{\varepsilon}-2 \gamma_{2} \geq \bar{r}_{\varepsilon}$, where we have used (29). This yields (41), which proves (40).

On the other hand, (38) yields

$$
\frac{f_{n_{k}}\left(u_{k, \varepsilon}\right)-f_{n_{k}}(w)}{d\left(u_{k, \varepsilon}, w\right)}<\varepsilon, \quad \forall w \in \overline{\left[F>\bar{r}_{\varepsilon}\right]} \backslash\left\{u_{k, \varepsilon}\right\} .
$$

Taking (40) into account, we can pass to lim sup as $w \rightarrow u_{k, \mathcal{\varepsilon}}$ in (42) to obtain

$$
\limsup _{w \rightarrow u_{k, \varepsilon}} \frac{f_{n_{k}}\left(u_{k, \varepsilon}\right)-f_{n_{k}}(w)}{d\left(u_{k, \varepsilon}, w\right)} \leq \varepsilon,
$$

where the limsup is taken for $w \in X$ (using (41)). If $u_{k, \varepsilon}$ is not a local minimum of $f_{n_{k}}$, then inequality (43) means that $\left|\nabla f_{n_{k}}\right|\left(u_{k, \varepsilon}\right) \leq \varepsilon$, while if $u_{k, \varepsilon}$ is a local minimum of $f_{n_{k}}$, then we know that $\left|\nabla f_{n_{k}}\right|\left(u_{k, \varepsilon}\right)=0$. Hence (6) is proved. Since there is no loss of generality in taking $\varepsilon \in\left(0, \gamma_{1} / 2\right)$, the proof of the first part of the conclusion is complete.

For every fixed integer $\ell \geq 1$, applying the first part of the conclusion with $\varepsilon=1 / \ell$, we find a subsequence $\left\{f_{n_{k}}\right\}$ (depending on $\ell$ ) of $\left\{f_{n}\right\}$ and a number $k_{\ell} \in \mathbb{N}$ such that for all $k \geq k_{\ell}$ there exists $u_{k, \ell} \in \operatorname{dom}\left(f_{n_{k}}\right)$ satisfying

$$
\begin{aligned}
\alpha-\frac{1}{\ell^{2}}<f_{n_{k}}\left(u_{k, \ell}\right)<\alpha+\frac{1}{\ell^{2}}, \quad\left|\nabla f_{n_{k}}\right|\left(u_{k, \ell}\right) & \leq \frac{1}{\ell}, \\
F\left(u_{k, \ell}\right) & >\ell .
\end{aligned}
$$

In particular, we obtain a subsequence $\left\{f_{m_{\ell}}\right\}$ of $\left\{f_{n_{k}}\right\}$ (setting $\left.m_{\ell}=n_{k_{\ell}}\right)$ and $u_{\ell}:=u_{k_{\ell}, \ell} \in \operatorname{dom}\left(f_{m_{\ell}}\right)$ with the properties stated in the second part of the conclusion.

Proof of Corollary 4. Arguing by contradiction, suppose that the sequence $\left\{f_{n}\right\}$ is not $F$-coercive; that is, $\alpha:=\alpha^{F}\left(\left\{f_{n}\right\}\right)<$ $+\infty$. Combining this with hypothesis $\mathrm{H}^{F}\left(\left\{f_{n}\right\}\right)$, we infer that $\alpha \in \mathbb{R}$. With all the hypotheses of Theorem 1 being satisfied, we then obtain a subsequence $\left\{f_{m_{\ell}}\right\}$ of $\left\{f_{n_{k}}\right\}$ and $u_{\ell} \in \operatorname{dom}\left(f_{m_{\ell}}\right)$ satisfying

$$
\begin{array}{r}
f_{m_{\ell}}\left(u_{\ell}\right) \longrightarrow \alpha, \quad\left|\nabla f_{m_{\ell}}\right|\left(u_{\ell}\right) \longrightarrow 0, \quad F\left(u_{\ell}\right) \longrightarrow+\infty \\
\text { as } \ell \longrightarrow \infty .
\end{array}
$$

The first two convergences in (45) in conjunction with condition (PS) $)^{F}$ yield that the sequence $\left\{u_{\ell}\right\}$ is $F$-bounded, which contradicts the last convergence in (45).

We conclude this section with the proof of some assertions stated in Section 1. First, recall that

$$
\alpha^{F}(f)=\liminf _{F(v) \rightarrow+\infty} f(v)=\sup _{r \in \mathbb{R}} \inf _{[F>r]} f=\sup _{r \in \mathbb{R}} \inf _{[F>r]} f,
$$

where the last equality is true in view of Lemma 16(a).

Lemma 17. Let $X$ be a metric space and let $F: X \rightarrow \mathbb{R}$ satisfy $\mathrm{H}(F)$. Given $c \in \mathbb{R}$, for every $f: X \rightarrow \mathbb{R} \cup\{+\infty\}$ one has that $c=\alpha^{F}(f)$ if and only if (11) holds.
Proof. Suppose that $c=\alpha^{F}(f)$. Then, we see from (46) that if $a<c$ then there exists $r \in \mathbb{R}$ such that $\inf _{[F>r]} f>a$ (i.e., $[f \leq a] \subset[F \leq r])$, and if $a>c$ then $\inf _{[F>r]} f<a$ for all $r \in \mathbb{R}$ (i.e., for every $r \in \mathbb{R}$ there exists $v_{r} \in[f \leq a] \backslash[F \leq r]$ ).

Conversely, suppose that $c$ satisfies (11). From the first part of (11), we have that if $a<c$, then there exists $r \in \mathbb{R}$ such that $[F>r] \subset[f>a]$. It follows that $\alpha^{F}(f) \geq \inf _{[F>r]} f \geq a$ (see (46)), whence $\alpha^{F}(f) \geq c$. From the second part of (11), we have that if $a>c$, then for every $r \in \mathbb{R}$, there exists $v_{r} \in[F>$ $r]$ such that $f\left(v_{r}\right) \leq a$; hence $\inf _{[F>r]} f \leq a$. We conclude that $\alpha^{F}(f) \leq a$ for all $a>c$, whence $\alpha^{F}(f) \leq c$.

Lemma 18. Let $X$ be a metric space and let $F: X \rightarrow \mathbb{R}$ satisfy $\mathrm{H}(F)$. Then for every $f: X \rightarrow \mathbb{R} \cup\{+\infty\}$ one has that $\alpha^{F}(f)>$ $-\infty$ if and only if $[F>b] \subset[f>a]$ for some $a, b \in \mathbb{R}$.

Proof. Suppose that $\alpha^{F}(f)>-\infty$. Then, by (46), there exist $a, b \in \mathbb{R}$ with $a<\alpha^{F}(f)$ such that $\inf _{[F>b]} f>a$; that is, $[F>b] \subset[f>a]$. Conversely, if $[F>b] \subset[f>a]$ for some $a, b \in \mathbb{R}$, then $\alpha^{F}(f) \geq \inf _{[F>b]} f \geq a>-\infty$ (see (46)).

Lemma 19. Let $X$ be a metric space, let $F: X \rightarrow \mathbb{R}$ satisfy $\mathrm{H}(F)$, and let $f, f_{n}: X \rightarrow \mathbb{R} \cup\{+\infty\}(n \in \mathbb{N})$ satisfy $\mathrm{H}^{F}\left(\left\{f_{n}\right\}, f\right)$. Then

$$
\alpha^{F}\left(\left\{f_{n}\right\}\right)\left(=\lim _{r \rightarrow+\infty} \liminf _{n \rightarrow \infty} \frac{\inf }{[F>r]} f_{n}\right)=\alpha^{F}(f) .
$$

Proof. Fix $r \geq r_{0}$, with $r_{0}$ given in $\mathrm{H}^{F}\left(\left\{f_{n}\right\}, f\right)$, and let $u \in$ $\operatorname{dom}(f) \cap \overline{[F>r]}$. By $\mathrm{H}^{F}\left(\left\{f_{n}\right\}, f\right)(\mathrm{i})$, there exists a sequence $\left\{u_{n}\right\}$ such that $u_{n} \rightarrow u$ and $f_{n}\left(u_{n}\right) \rightarrow f(u)$ as $n \rightarrow \infty$. By Lemma 16, we have $u \in \overline{[F>r]} \subset \operatorname{int}\left[F>r-2 \gamma_{2}\right]$. Then $u_{n} \in\left[F>r-2 \gamma_{2}\right]$ for all $n \in \mathbb{N}$ sufficiently large, say $n \geq n_{0}$. It follows that

$$
\frac{\inf }{\left[F>r-2 \gamma_{2}\right]} f_{n} \leq f_{n}\left(u_{n}\right), \quad \forall n \geq n_{0} .
$$

Passing to $\lim \inf$ as $n \rightarrow \infty$ and using $\mathrm{H}^{F}\left(\left\{f_{n}\right\}, f\right)$ (ii), we infer that

$$
\frac{\inf f}{[F>r]} \leq \liminf _{n \rightarrow \infty} \frac{\inf }{\left[F>r-2 \gamma_{2}\right]} f_{n} \leq \lim _{n \rightarrow \infty} f_{n}\left(u_{n}\right)=f(u) .
$$

Since this inequality holds for every $u \in \overline{[F>r]}$, we obtain

$$
\liminf _{n \rightarrow \infty} \frac{\inf }{\left[F>r-2 \gamma_{2}\right]} f_{n}=\frac{\inf }{[F>r]} f .
$$

Since $r \geq r_{0}$ is arbitrary, letting $r \rightarrow+\infty$ establishes the lemma.

\section{Special Cases and Further Remarks}

3.1. Case of the Existence of Limit in $\alpha^{F}\left(\left\{f_{n}\right\}\right)$. Let $X$ be a Banach space, let $F: X \rightarrow \mathbb{R}$ be a function satisfying $\mathrm{H}(F)$, and let $f_{n}: X \rightarrow \mathbb{R} \cup\{+\infty\}(n \in \mathbb{N})$ be a sequence of lower semicontinuous functions which are not identically $+\infty$. We assume the following. 
$\widetilde{\mathrm{H}}^{F}\left(\left\{f_{n}\right\}\right):$ there exists $r_{0} \in \mathbb{R}$ such that for all $r>r_{0}$, $\lim _{n \rightarrow \infty} \inf _{\overline{[F>r]}} f_{n}$ exists.

Note that $\widetilde{\mathrm{H}}^{F}\left(\left\{f_{n}\right\}\right)$ is satisfied by all the functions in Example 12(a), (b), and (c). We consider another notion of Palais-Smale condition relative to $F$.

Definition 20. Let $f_{n}: X \rightarrow \mathbb{R} \cup\{+\infty\}(n \in \mathbb{N})$ be lower semicontinuous functions which are not identically $+\infty$. We say that the sequence $\left\{f_{n}\right\}$ satisfies the generalized PalaisSmale condition relative to $F$ (condition (gPS) ${ }^{F}$, for short) if there exists a subsequence $\left\{f_{n_{k}}\right\}$ of $\left\{f_{n}\right\}$ such that whenever $\left\{u_{k}\right\} \subset X$ is a sequence such that $\left\{f_{n_{k}}\left(u_{k}\right)\right\}$ is bounded and $\left|\nabla f_{n_{k}}\right|\left(u_{k}\right) \rightarrow 0$ as $k \rightarrow \infty$, then $\left\{u_{k}\right\}$ is $F$-bounded.

Remark 21. Condition $(\mathrm{gPS})^{F}$ is more general than condition $(\mathrm{PS})^{F}$ of Definition 2.

Corollary 22. Assume that $\mathrm{H}^{F}\left(\left\{f_{n}\right\}\right)$ and $\widetilde{\mathrm{H}}^{F}\left(\left\{f_{n}\right\}\right)$ hold.

(i) Assume $\alpha^{F}\left(\left\{f_{n}\right\}\right)<+\infty$. Then for every $\varepsilon>0$, there exists $n_{\varepsilon} \in \mathbb{N}$ such that for each $n \geq n_{\varepsilon}$ one finds $u_{n, \varepsilon} \in$ $\operatorname{dom}\left(f_{n}\right)$ satisfying

$$
\begin{gathered}
\alpha^{F}\left(\left\{f_{n}\right\}\right)-\varepsilon^{2}<f_{n}\left(u_{n, \varepsilon}\right)<\alpha^{F}\left(\left\{f_{n}\right\}\right)+\varepsilon^{2}, \\
\left|\nabla f_{n}\right|\left(u_{n, \varepsilon}\right) \leq \varepsilon, \quad F\left(u_{n, \varepsilon}\right)>\frac{1}{\varepsilon} .
\end{gathered}
$$

In particular, there exists $u_{n} \in \operatorname{dom}\left(f_{n}\right)(n \in \mathbb{N})$ satisfying

$$
\begin{gathered}
f_{n}\left(u_{n}\right) \longrightarrow \alpha^{F}\left(\left\{f_{n}\right\}\right), \quad\left|\nabla f_{n}\right|\left(u_{n}\right) \longrightarrow 0, \\
F\left(u_{n}\right) \longrightarrow+\infty \quad \text { as } n \longrightarrow \infty .
\end{gathered}
$$

(ii) Assume that $\left\{f_{n}\right\}$ satisfies condition (gPS) ${ }^{F}$ and that $\sup _{X} F=+\infty$. Then the sequence $\left\{f_{n}\right\}$ is $F$-coercive.

Proof. (i) We argue as in the proof of Theorem 1 noting that, due to the assumption, in place of (27) and (28) we have

$$
\alpha-\varepsilon^{2}<\inf _{[F>r]} f_{n}<\alpha+\varepsilon^{2}, \quad \forall n \geq n_{r, \varepsilon}
$$

and choosing $n_{\varepsilon}=\max \left\{n_{\bar{r}_{\varepsilon}, \varepsilon}, n_{2 \bar{r}_{\varepsilon}, \varepsilon}\right\}$.

(ii) Arguing by contradiction, suppose that the sequence $\left\{f_{n}\right\}$ is not $F$-coercive; that is, $\alpha:=\alpha^{F}\left(\left\{f_{n}\right\}\right)<+\infty$; thus $\alpha \epsilon$ $\mathbb{R}\left(\right.$ see $\left.\mathrm{H}^{F}\left(\left\{f_{n}\right\}\right)\right)$. Then, by part (i), we can find $u_{n} \in \operatorname{dom}\left(f_{n}\right)$ satisfying (52). Let $\left\{f_{n_{k}}\right\}$ be the subsequence of $\left\{f_{n}\right\}$ that satisfies condition $(\mathrm{gPS})^{F}$. Then the convergences $f_{n_{k}}\left(u_{n_{k}}\right) \rightarrow$ $\alpha$ and $\left|\nabla f_{n_{k}}\right|\left(u_{n_{k}}\right) \rightarrow 0$ yield that $\left\{u_{n_{k}}\right\}$ is F-bounded, which contradicts the third convergence in (52).
3.2. Case of Functionals with Special Structure. Let $(X,\|\cdot\|)$ be a Banach space, $F: X \rightarrow \mathbb{R}$ be a function satisfying $\mathrm{H}(F)$, and $f_{n}: X \rightarrow \mathbb{R} \cup\{+\infty\}(n \in \mathbb{N})$ be of the form

$$
f_{n}=\Phi_{n}+\Psi_{n}
$$

with $\Phi_{n}: X \rightarrow \mathbb{R}$ locally Lipschitz and $\Psi_{n}: X \rightarrow \mathbb{R} \cup\{+\infty\}$ convex, lower semicontinuous, not identically $+\infty$. In this setting we consider an appropriate version of Palais-Smale condition (see Motreanu and Panagiotopoulos [5, Chapter 3]).

Definition 23. The sequence of functionals $\left\{f_{n}\right\}$ as in (54) satisfies the Palais-Smale condition in the sense of Motreanu and Panagiotopoulos relative to $F$ (condition $\left(\mathrm{PS}^{+}\right)^{F}$ for short) if whenever $\left\{f_{n_{k}}\right\}$ is a subsequence of $\left\{f_{n}\right\}$ and $\left\{u_{k}\right\} \subset X$ is a sequence such that $\left\{f_{n_{k}}\left(u_{k}\right)\right\}$ is bounded and for which there exists a sequence $\left\{\varepsilon_{k}\right\} \subset \mathbb{R}^{+}, \varepsilon_{k} \downarrow 0$, such that

$$
\begin{aligned}
& \Phi_{n_{k}}^{0}\left(u_{k} ; v-u_{k}\right)+\Psi_{n_{k}}(v)-\Psi_{n_{k}}\left(u_{k}\right) \\
& \geq-\varepsilon_{k}\left\|v-u_{k}\right\|, \quad \forall v \in X, \quad \forall k \geq 1,
\end{aligned}
$$

then $\left\{u_{k}\right\}$ is $F$-bounded.

Hereafter, the notation $\Phi^{0}(u ; v)$ stands for the generalized directional derivative of a locally Lipschitz functional $\Phi$ : $X \rightarrow \mathbb{R}$ at the point $u \in X$ in the direction $v \in X$ (see Clarke [6]) given by

$$
\Phi^{0}(u ; v)=\limsup _{\substack{w \rightarrow u \\ t \downarrow 0}} \frac{1}{t}(\Phi(w+t v)-\Phi(w))
$$

Remark 24. Condition $\left(\mathrm{PS}^{+}\right)^{F}$ in the above definition generalizes the Palais-Smale conditions of Chang [7] (for the case where $\Phi_{n} \equiv \Phi$ is locally Lipschitz, $\Psi=0$, and $\left.F=\|\cdot\|\right)$ and Szulkin [8] (for the case where $\Phi_{n} \equiv \Phi \in C^{1}(X, \mathbb{R})$, $\Psi_{n} \equiv \Psi$ is lower semicontinuous, convex, not identically $+\infty$, and $F=\|\cdot\|)$.

Lemma 25. (a) Let $\Phi: X \rightarrow \mathbb{R}$ be a locally Lipschitz functional, let $\Psi: X \rightarrow \mathbb{R} \cup\{+\infty\}$ be a convex, lower semicontinuous function which is not identically $+\infty$, and let $f=\Phi+\Psi$. Then

$$
\begin{aligned}
& \Phi^{0}(u ; v-u)+\Psi(v)-\Psi(u) \\
& \quad \geq-|\nabla f|(u)\|u-v\|, \quad \forall u, v \in X .
\end{aligned}
$$

(b) For a sequence $\left\{f_{n}\right\}$ as in Definition 23, one has

$\left(\mathrm{PS}^{+}\right)^{F}$ (of Definition 23) $\Longrightarrow(\mathrm{PS})^{F}$ (of Definition 2). 
Proof. (a) Using the convexity of $\Psi$, for every $u, v \in X, v \neq u$, we have

$$
\begin{aligned}
& \frac{\Phi^{0}(u ; v-u)}{\|u-v\|}+\frac{\Psi(v)-\Psi(u)}{\|u-v\|} \\
& \geq \limsup _{t \downarrow 0} \frac{\Phi(u+t(v-u))-\Phi(u)}{t\|v-u\|} \\
& \quad+\lim _{t \downarrow 0} \frac{\Psi(u+t(v-u))-\Psi(u)}{t\|u-v\|} \\
& \geq \liminf _{w \rightarrow u}\left(\frac{\Phi(w)-\Phi(u)}{\|w-u\|}+\frac{\Psi(w)-\Psi(u)}{\|w-u\|}\right) \\
& =-\limsup _{w \rightarrow u} \frac{f(u)-f(w)}{\|u-w\|} .
\end{aligned}
$$

If $u$ is not a local minimum of $f$, then the desired inequality follows. If $u$ is a local minimum of $f$, then it is a critical point of $f$ in the sense of Motreanu-Panagiotopoulos [5, Definition 3.1]; that is, $\Phi^{0}(u ; v-u)+\Psi(v)-\Psi(u) \geq 0$ for all $v \in X$, and so again we are done.

(b) This is an immediate consequence of part (a).

Corollary 26. Let $X$ be a Banach space and let $F: X \rightarrow \mathbb{R}$ satisfy $\mathrm{H}(F)$. Let $f_{n}=\Phi_{n}+\Psi_{n}: X \rightarrow \mathbb{R} \cup\{+\infty\}(n \in \mathbb{N})$ as in (54). Assume that $\mathrm{H}^{F}\left(\left\{f_{n}\right\}\right)$ holds.

(i) Assume $\alpha^{F}\left(\left\{f_{n}\right\}\right)<+\infty$. Then, for every $\varepsilon>0$, there exist a subsequence $\left\{f_{n_{k}}\right\}$ (depending on $\varepsilon$ ) of $\left\{f_{n}\right\}$ and a number $k_{\varepsilon} \in \mathbb{N}$ such that for each $k \geq k_{\varepsilon}$ one finds $u_{k, \varepsilon} \in \operatorname{dom}\left(f_{n_{k}}\right)$ satisfying

$\alpha^{F}\left(\left\{f_{n}\right\}\right)-\varepsilon^{2}<f_{n_{k}}\left(u_{k, \varepsilon}\right)<\alpha^{F}\left(\left\{f_{n}\right\}\right)+\varepsilon^{2}, \quad F\left(u_{k, \varepsilon}\right)>\frac{1}{\varepsilon}$,

$\Phi_{n_{k}}^{0}\left(u_{k, \varepsilon} ; v-u_{k, \varepsilon}\right)+\Psi_{n_{k}}(v)-\Psi_{n_{k}}\left(u_{k, \varepsilon}\right)$

$\geq-\varepsilon\left\|v-u_{k, \varepsilon}\right\|, \quad \forall v \in X, \forall k \geq 1$.

(ii) Assume that $\left\{f_{n}\right\}$ satisfies condition $\left(\mathrm{PS}^{+}\right)^{F}$ and that $\sup _{X} F=+\infty$. Then the sequence $\left\{f_{n}\right\}$ is $F$-coercive.

Proof. Part (i) follows from Theorem 1 by using Lemma 25 (a), while part (ii) follows from Corollary 4 by using Lemma 25(b).

Remark 27. When all the terms of the sequence $\left\{f_{n}\right\}$ coincide (and $F=\|\cdot\|$ ), an extension of Corollary 26 has been obtained in Motreanu et al. [9] by means of a general Palais-Smale condition incorporating the Palais-Smale conditions in the sense of Cerami [10] and Zhong [11].

3.3. Case of Galerkin Approximations. Let $(X,\|\cdot\|)$ be a Banach space, let $\left\{X_{n}\right\}$ be a sequence of closed vector subspaces of $X$ (not necessarily increasing) such that $\overline{\bigcup_{n=1}^{\infty} X_{n}}=X$, and let $F: X \rightarrow \mathbb{R}$ be a function satisfying $\mathrm{H}(F)$. Let $f \in C^{1}(X, \mathbb{R})$ and let

$$
f_{n}:=\left.f\right|_{X_{n}} \in C^{1}\left(X_{n} ; \mathbb{R}\right), \quad \forall n \in \mathbb{N}
$$

We consider the following Palais-Smale condition (see Li and Willem [12]).

Definition 28. The function $f \in C^{1}(X, \mathbb{R})$ satisfies the PalaisSmale condition in the sense of Li-Willem relative to $F$ (condition $\left(\mathrm{PS}^{*}\right)^{F}$ for short) if every sequence $\left\{u_{n}\right\} \subset X$ with $u_{n} \in X_{\alpha_{n}}, \alpha_{n} \rightarrow+\infty,\left\{f\left(u_{n}\right)\right\}$ bounded and $f_{\alpha_{n}}^{\prime}\left(u_{n}\right) \rightarrow 0$ is $F$-bounded. by

For all $n \in \mathbb{N}$, define the functions $\tilde{f}_{n}: X \rightarrow \mathbb{R} \cup\{+\infty\}$

$$
\widetilde{f}_{n}(u)= \begin{cases}f(u), & \text { if } u \in X_{n} \\ +\infty, & \text { otherwise }\end{cases}
$$

It is clear that the functions $\tilde{f}_{n}$ are lower semicontinuous and not identically $+\infty$.

Lemma 29. (a) $\left|\nabla \tilde{f}_{n}\right|(u)=\left\|f_{n}^{\prime}(u)\right\|_{X_{n}^{*}}$ for all $u \in X_{n}$ and all $n \in \mathbb{N}$.

(b) $\left(\mathrm{PS}^{*}\right)^{F}$ (of Definition 28 for $\left.f \in C^{1}(X, \mathbb{R})\right) \Leftrightarrow(\mathrm{PS})^{F}$ (of Definition 2 for $\left.\left\{\tilde{f}_{n}\right\}\right)$.

Proof. (a) Using that $f_{n} \in C^{1}\left(X_{n}, \mathbb{R}\right)$, for every $u \in X_{n}$ which is not a local minimizer of $\widetilde{f}_{n}$ (equivalently, nor of $f_{n}$ ) we have

$$
\begin{aligned}
\left|\nabla \tilde{f}_{n}\right|(u) & =\limsup _{\substack{w \rightarrow u \\
w \in X_{n}}} \frac{\tilde{f}_{n}(u)-\tilde{f}_{n}(w)}{\|u-w\|} \\
& =\left|\nabla f_{n}\right|(u)=\left\|f_{n}^{\prime}(u)\right\|_{X_{n}^{*}}
\end{aligned}
$$

The case where $u \in X_{n}$ is a local minimizer of $\tilde{f}_{n}$ is straightforward.

(b) This easily follows from part (a).

Denote $\alpha_{f}^{F}:=\lim _{r \rightarrow+\infty} \liminf _{n \rightarrow \infty} \inf _{\overline{[F>r]} \cap X_{n}} f$.

Corollary 30. Let $X$ be a Banach space, let $\left\{X_{n}\right\}$ be a sequence of closed vector subspaces of $X$ such that $\overline{\bigcup_{n=1}^{\infty} X_{n}}=X$, and let $F: X \rightarrow \mathbb{R}$ be a function satisfying $\mathrm{H}(F)$. Let $f \in C^{1}(X, \mathbb{R})$ and $f_{n}:=\left.f\right|_{X_{n}}(n \in \mathbb{N})$. Assume that $\alpha_{f}^{F}>-\infty$.

(i) Assume $\alpha_{f}^{F}<+\infty$. Then, for every $\varepsilon>0$, there exist a subsequence $\left\{f_{n_{k}}\right\}$ (depending on $\varepsilon$ ) of $\left\{f_{n}\right\}$ and $a$ number $k_{\varepsilon} \in \mathbb{N}$ such that for each $k \geq k_{\varepsilon}$ one finds $u_{k, \varepsilon} \in X_{n_{k}}$ satisfying

$$
\begin{aligned}
& \alpha_{f}^{F}-\varepsilon^{2}<f\left(u_{k, \varepsilon}\right)<\alpha_{f}^{F}+\varepsilon^{2}, \\
& \left\|f_{n_{k}}^{\prime}\left(u_{k, \varepsilon}\right)\right\|_{X_{n_{k}}^{*}} \leq \varepsilon, \quad F\left(u_{k, \varepsilon}\right)>\frac{1}{\varepsilon} .
\end{aligned}
$$

(ii) Assume that $f$ satisfies condition $\left(P S^{*}\right)^{F}$ and that $\sup _{X} F=+\infty$. Then $\alpha_{f}^{F}=+\infty$.

Proof. Part (i) follows by applying Theorem 1 to the sequence $\left\{\tilde{f}_{n}\right\}$ in (62), noting that $\alpha^{F}\left(\left\{\tilde{f}_{n}\right\}\right)=\alpha_{f}^{F}$ and using 
Lemma 29(a). To prove part (ii), apply Corollary 4 to $\left\{\tilde{f}_{n}\right\}$ and use Lemma 29(b).

3.4. Case of a Constant Sequence of Functions. When all the terms of the sequence $\left\{f_{n}\right\}$ coincide, say $f_{n}=f$, for all $n$, Theorem 1 and Corollary 4 yield the following.

Corollary 31. Let $X$ be a complete metric space and let $F$ : $X \rightarrow \mathbb{R}$ satisfy $\mathrm{H}(F)$. Let $f: X \rightarrow \mathbb{R} \cup\{+\infty\}$ be a lower semicontinuous function with the property

$$
\alpha^{F}(f):=\liminf _{F(v) \rightarrow+\infty} f(v)\left(=\lim _{r \rightarrow+\infty} \inf _{[F>r]} f\right)>-\infty
$$

(i) If $\alpha^{F}(f)<+\infty$, then for every $\varepsilon>0$ there exists $u_{\varepsilon} \in$ $\operatorname{dom}(f)$ such that

$$
\begin{gathered}
\alpha^{F}(f)-\varepsilon^{2}<f\left(u_{\varepsilon}\right)<\alpha^{F}(f)+\varepsilon^{2}, \\
|\nabla f|\left(u_{\varepsilon}\right) \leq \varepsilon, \quad F\left(u_{\varepsilon}\right)>\frac{1}{\varepsilon} .
\end{gathered}
$$

(ii) Assume that $f$ satisfies the following Palais-Smale condition: every sequence $\left\{u_{k}\right\} \subset X$ such that $\left\{f\left(u_{k}\right)\right\}$ is bounded and $|\nabla f|\left(u_{k}\right) \rightarrow 0$ as $k \rightarrow \infty$ is F-bounded. Assume also that $\sup _{X} F=+\infty$. Then $f$ is F-coercive; that is, $\alpha^{F}(f)=+\infty$, or, equivalently, $f(v) \rightarrow+\infty$ as $F(v) \rightarrow+\infty$.

Proof. Part (i) follows from Theorem 1 applied with $f_{n}=f$ for all $n \in \mathbb{N}$ (note that $\alpha^{F}(f)=\alpha^{F}(\{f\})$ ), while part (ii) follows from Corollary 4 in the same way.

Remark 32. (a) The condition that $\alpha^{F}(f) \in \mathbb{R}$ ensures that the function $f$ is not identically $+\infty$ and that the sets $\{v \in X$ : $F(v)>r$ \} are nonempty for all $r \in \mathbb{R}$.

(b) In Motreanu et al. [13], results concerning the asymptotic behavior as in Corollary 31(i) and the F-coercivity as in Corollary 31(ii) are given in the more general setting of a metric space endowed with a quasiorder $\leq$ and of $\leq$ lower semicontinuous functions, by means of an appropriate notion of strong slope. Corollary 31 can be obtained from [13, Theorems 6.1, and 6.2] in the case where the quasiorder is the trivial one.

We apply Corollary 31 to the special situation $F=d(\cdot, A)$ (see Example 13(a)).

Corollary 33. Let $X$ be a complete metric space, let $A$ be a nonempty subset of $X$, and let $f: X \rightarrow \mathbb{R} \cup\{+\infty\}$ be a lower semicontinuous function with the property

$$
\alpha^{d(\cdot, A)}(f):=\liminf _{d(v, A) \rightarrow+\infty} f(v)>-\infty
$$

(i) If $\alpha^{d(\cdot, A)}(f)<+\infty$, then for every $\varepsilon>0$ there is $u_{\varepsilon} \epsilon$ $\operatorname{dom}(f)$ such that

$$
\begin{gathered}
\alpha^{d(\cdot, A)}(f)-\varepsilon^{2}<f\left(u_{\varepsilon}\right)<\alpha^{d(\cdot, A)}(f)+\varepsilon^{2}, \\
|\nabla f|\left(u_{\varepsilon}\right) \leq \varepsilon, \quad d\left(u_{\varepsilon}, A\right)>\frac{1}{\varepsilon} .
\end{gathered}
$$

(ii) Assume that $f$ satisfies the following Palais-Smale condition: every sequence $\left\{u_{k}\right\} \subset X$ such that $\left\{f\left(u_{k}\right)\right\}$ is bounded and $|\nabla f|\left(u_{k}\right) \rightarrow 0$ as $k \rightarrow \infty$ satisfies that $\left\{d\left(u_{k}, A\right)\right\}$ is bounded. If $X \backslash A$ is unbounded, then $f$ is coercive in the sense that $f(v) \rightarrow+\infty$ as $d(v, A) \rightarrow+\infty$.

Proof. This readily follows from Corollary 31 applied to $F=$ $d(\cdot, A)$.

Remark 34. In the case where $A$ is bounded, the expression $\alpha^{d(\cdot, A)}(f)$ in Corollary 33 and the coercivity property in (ii) do not depend on the set $A \subset X$.

In particular, we can consider $A=\left\{u_{0}\right\}$, for some $u_{0} \in X$. If $X$ is a Banach space and $u_{0}=0$, then Corollary 33 becomes the following.

Corollary 35. Let $(X,\|\cdot\|)$ be a Banach space and let $f: X \rightarrow$ $\mathbb{R} \cup\{+\infty\}$ be a lower semicontinuous function with the property $\alpha^{\|\cdot\|}(f):=\liminf _{\|v\| \rightarrow+\infty} f(v)>-\infty$.

(i) If $\alpha^{\|\cdot\|}(f)<+\infty$, then for every $\varepsilon>0$ there exists $u_{\varepsilon} \epsilon$ $\operatorname{dom}(f)$ such that

$$
\begin{gathered}
\alpha^{\|\cdot\|}(f)-\varepsilon^{2}<f\left(u_{\varepsilon}\right)<\alpha^{\|\cdot\|}(f)+\varepsilon^{2}, \\
|\nabla f|\left(u_{\varepsilon}\right) \leq \varepsilon, \quad\left\|u_{\varepsilon}\right\|>\frac{1}{\varepsilon} .
\end{gathered}
$$

(ii) Assume that $f$ satisfies the following Palais-Smale condition: every sequence $\left\{u_{k}\right\} \subset X$ such that $\left\{f\left(u_{k}\right)\right\}$ is bounded and $|\nabla f|\left(u_{k}\right) \rightarrow 0$ as $k \rightarrow \infty$ is bounded. Then $f$ is coercive in the sense that $f(v) \rightarrow+\infty$ as $\|v\| \rightarrow+\infty$.

Remark 36. Corollary 35 extends the corresponding result in the smooth case, that is, for $f$ of class $C^{1}$ (see Brezis and Nirenberg [14]).

3.5. Case Where the Limits in $\alpha^{F}\left(\left\{f_{n}\right\}\right)$ Are Interchanged. It is natural to ask what happens when the expression $\alpha^{F}\left(\left\{f_{n}\right\}\right)$ in $\mathrm{H}^{F}\left(\left\{f_{n}\right\}\right)$ is replaced with

$$
\begin{aligned}
\tilde{\alpha}^{F}\left(\left\{f_{n}\right\}\right):= & \lim _{n \rightarrow \infty} \liminf _{F(v) \rightarrow+\infty} f_{n}(v) \stackrel{\text { def }}{=} \lim _{n \rightarrow \infty} \lim _{r \rightarrow+\infty} \frac{\inf }{[F>r]} f_{n} \\
& =\lim _{n \rightarrow \infty} \sup _{r \in \mathbb{R}} \inf f_{n},
\end{aligned}
$$

that is, when the limits in $\mathrm{H}^{F}\left(\left\{f_{n}\right\}\right)$ are interchanged (we work directly with a sequence $\left\{f_{n}\right\}$ for which the limit as $n \rightarrow \infty$ in (70) exists in place of considering lim inf). 
Proposition 37. Let $X$ be a complete metric space, let $F: X \rightarrow$ $\mathbb{R}$ be a function satisfying $\mathrm{H}(F)$, and let $f_{n}: X \rightarrow \mathbb{R} \cup\{+\infty\}$ $(n \in \mathbb{N})$ be a sequence of lower semicontinuous functions with the property $\tilde{\alpha}^{F}\left(\left\{f_{n}\right\}\right)>-\infty$.

(i) Assume $\tilde{\alpha}^{F}\left(\left\{f_{n}\right\}\right)<+\infty$. Then for every $\varepsilon>0$, there exists $n_{\varepsilon} \in \mathbb{N}$ such that for each $n \geq n_{\varepsilon}$ one finds $u_{n, \varepsilon} \in$ $\operatorname{dom}\left(f_{n}\right)$ satisfying

$$
\begin{gathered}
\tilde{\alpha}^{F}\left(\left\{f_{n}\right\}\right)-\varepsilon^{2}<f_{n}\left(u_{n, \varepsilon}\right)<\widetilde{\alpha}^{F}\left(\left\{f_{n}\right\}\right)+\varepsilon^{2}, \\
\left|\nabla f_{n}\right|\left(u_{n, \varepsilon}\right) \leq \varepsilon, \quad F\left(u_{n, \varepsilon}\right)>\frac{1}{\varepsilon} .
\end{gathered}
$$

(ii) Assume that $\left\{f_{n}\right\}$ satisfies condition (gPS) $)^{F}$ (see Definition 20) and that $\sup _{X} F=+\infty$. Then $\tilde{\alpha}^{F}\left(\left\{f_{n}\right\}\right)=+\infty$.

Proof. (i) We denote $\tilde{\alpha}:=\widetilde{\alpha}^{F}\left(\left\{f_{n}\right\}\right) \in \mathbb{R}$. Fix $\varepsilon>0$. By (70), there exists $n_{\varepsilon} \in \mathbb{N}$ such that

$$
\tilde{\alpha}-\frac{\varepsilon^{2}}{2}<\lim _{r \rightarrow+\infty} \inf _{[F>r]} f_{n}<\tilde{\alpha}+\frac{\varepsilon^{2}}{2}, \quad \forall n \geq n_{\varepsilon} .
$$

Denote $\alpha_{n}:=\lim _{r \rightarrow+\infty} \inf _{\overline{[F>r]}} f_{n}$. In view of (72) we have $\alpha_{n} \in \mathbb{R}$ for all $n \geq n_{\varepsilon}$. Then, for each $n \geq n_{\varepsilon}$ we can apply Corollary 31(i) to the function $f:=f_{n}$ and the number $\widetilde{\varepsilon}:=$ $\varepsilon / \sqrt{2}$. Thus, we find $u_{n, \varepsilon} \in \operatorname{dom}\left(f_{n}\right)$ such that

$$
\begin{gathered}
\tilde{\alpha}-\varepsilon^{2}<\alpha_{n}-\frac{\varepsilon^{2}}{2}<f_{n}\left(u_{n, \varepsilon}\right)<\alpha_{n}+\frac{\varepsilon^{2}}{2}<\tilde{\alpha}+\varepsilon^{2}, \\
\left|\nabla f_{n}\right|\left(u_{n, \varepsilon}\right) \leq \frac{\varepsilon}{\sqrt{2}}<\varepsilon, \quad F\left(u_{n, \varepsilon}\right)>\frac{\sqrt{2}}{\varepsilon}>\frac{1}{\varepsilon} .
\end{gathered}
$$

(ii) Arguing by contradiction, suppose that $\widetilde{\alpha}:=$ $\widetilde{\alpha}^{F}\left(\left\{f_{n}\right\}\right) \in \mathbb{R}$. Let a subsequence of $\left\{f_{n}\right\}$, denoted again by $\left\{f_{n}\right\}$, with the property in condition (gPS) ${ }^{F}$. Applying part (i) of the proposition to the subsequence $\left\{f_{n}\right\}$ and $\varepsilon=1 / k(k \in$ $\mathbb{N})$, we find a number $n_{k} \in \mathbb{N}$ and an element $u_{k} \in \operatorname{dom}\left(f_{n_{k}}\right)$ with the properties

$$
\begin{array}{r}
\tilde{\alpha}-\frac{1}{k^{2}}<f_{n_{k}}\left(u_{k}\right)<\tilde{\alpha}+\frac{1}{k^{2}}, \quad\left|\nabla f_{n_{k}}\right|\left(u_{k}\right) \leq \frac{1}{k}, \\
F\left(u_{k}\right)>k .
\end{array}
$$

Consequently, we have $f_{n_{k}}\left(u_{k}\right) \rightarrow \tilde{\alpha},\left|\nabla f_{n_{k}}\right|\left(u_{k}\right) \rightarrow 0$, and $F\left(u_{k}\right) \rightarrow+\infty$ as $k \rightarrow \infty$. The first two convergences in conjunction with condition (gPS) ${ }^{F}$ yield that the sequence $\left\{u_{k}\right\}$ is $F$-bounded, which contradicts the third convergence.

Remark 38. The above proof shows that Proposition 37(i) is a consequence of Corollary 31(i). On the other hand, Corollary 31(i) can be obtained from Proposition 37(i) by taking $f_{n}=f$, for all $n \in \mathbb{N}$. So, Proposition 37(i) and Corollary 31(i) are conceptually equivalent, in the sense that

$$
\text { Corollary 31(i) } \Longrightarrow \text { Proposition 37(i) } \Longrightarrow \text { Corollary 31(i) . }
$$

Thus our main result, Theorem 1 (involving the expression $\alpha^{F}\left(\left\{f_{n}\right\}\right)$ in $\mathrm{H}^{F}\left(\left\{f_{n}\right\}\right)$ is more general than Proposition 37(i) (involving the expression $\widetilde{\alpha}^{F}\left(\left\{f_{n}\right\}\right)$ in (70)).

\section{Acknowledgment}

V. V. Motreanu is supported by a Marie Curie Intra-European Fellowship for Career Development within the European Community's 7th Framework Program (Grant Agreement no. PIEF-GA-2010-274519).

\section{References}

[1] E. De Giorgi, A. Marino, and M. Tosques, "Problems of evolution in metric spaces and maximal decreasing curve," Atti della Accademia Nazionale dei Lincei. Rendiconti, Classe di Scienze Fisiche, Matematiche e Naturali, vol. 68, no. 3, pp. 180-187, 1980.

[2] J. N. Corvellec, "A note on coercivity of lower semicontinuous functions and nonsmooth critical point theory," Serdica Mathematical Journal, vol. 22, no. 1, pp. 57-68, 1996.

[3] D. Motreanu and V. V. Motreanu, "Coerciveness property for a class of non-smooth functionals," Zeitschrift für Analysis und ihre Anwendungen, vol. 19, no. 4, pp. 1087-1093, 2000.

[4] I. Ekeland, "On the variational principle," Journal of Mathematical Analysis and Applications, vol. 47, pp. 324-353, 1974.

[5] D. Motreanu and P. D. Panagiotopoulos, Minimax Theorems and Qualitative Properties of the Solutions of Hemivariational Inequalities, vol. 29 of Nonconvex Optimization and Its Applications, Kluwer Academic Publishers, Dordrecht, The Netherlands, 1999.

[6] F. H. Clarke, Optimization and Nonsmooth Analysis, vol. 5 of Classics in Applied Mathematics, SIAM, Philadelphia, Pa, USA, 2nd edition, 1990.

[7] K. C. Chang, "Variational methods for nondifferentiable functionals and their applications to partial differential equations," Journal of Mathematical Analysis and Applications, vol. 80, no. 1, pp. 102-129, 1981.

[8] A. Szulkin, "Minimax principles for lower semicontinuous functions and applications to nonlinear boundary value problems," Annales de l'Institut Henri Poincaré, Analyse Non Linéaire, vol. 3, no. 2, pp. 77-109, 1986.

[9] D. Motreanu, V. V. Motreanu, and D. Paşca, "A version of Zhong's coercivity result for a general class of nonsmooth functionals," Abstract and Applied Analysis, vol. 7, no. 11, pp. 601$612,2002$.

[10] G. Cerami, "An existence criterion for the critical points on unbounded manifolds," Istituto Lombardo, Accademia di Scienze e Lettere. Rendiconti, vol. 112, no. 2, pp. 332-336, 1978.

[11] C. K. Zhong, "A generalization of Ekeland's variational principle and application to the study of the relation between the weak P.S. condition and coercivity," Nonlinear Analysis: Theory, Methods \& Applications, vol. 29, no. 12, pp. 1421-1431, 1997.

[12] S. J. Li and M. Willem, "Applications of local linking to critical point theory," Journal of Mathematical Analysis and Applications, vol. 189, no. 1, pp. 6-32, 1995.

[13] D. Motreanu, V. V. Motreanu, and M. Turinici, "Coerciveness property for conical nonsmooth functionals," Journal of Optimization Theory and Applications, vol. 145, no. 1, pp. 148-163, 2010.

[14] H. Brezis and L. Nirenberg, "Remarks on finding critical points," Communications on Pure and Applied Mathematics, vol. 44, no. 8-9, pp. 939-963, 1991. 


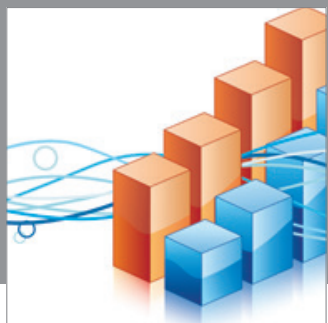

Advances in

Operations Research

mansans

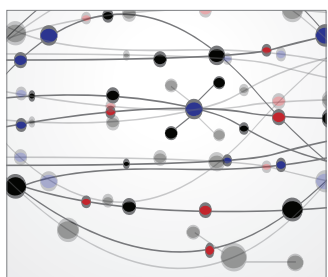

The Scientific World Journal
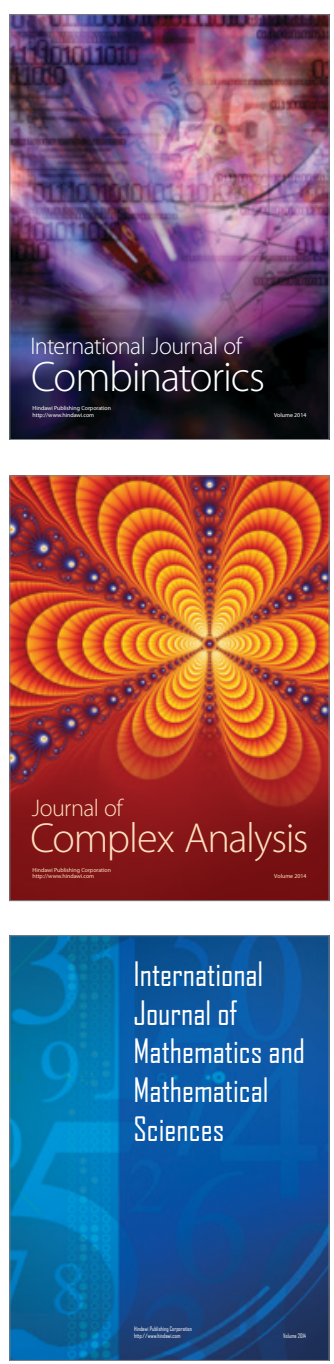
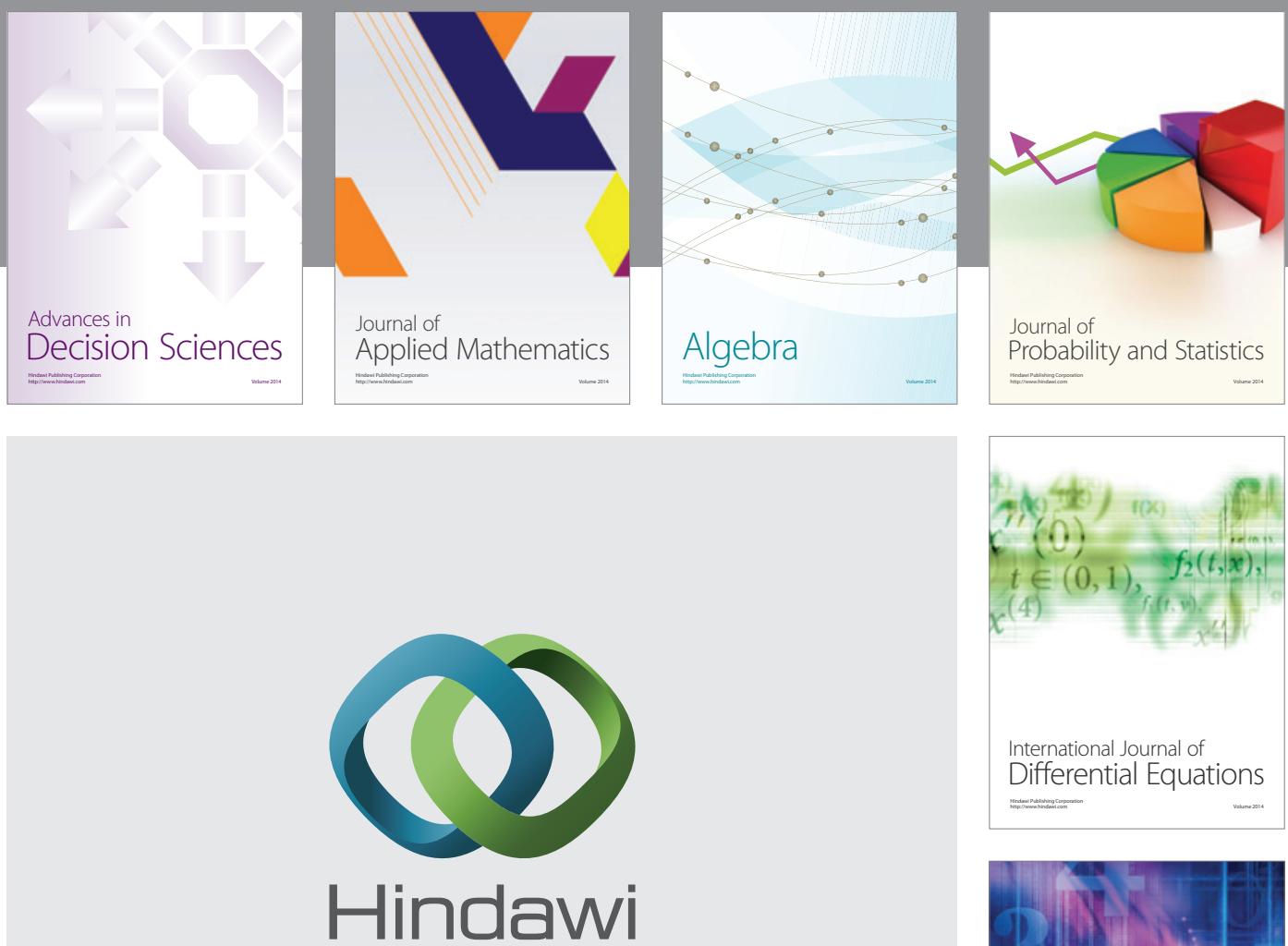

Submit your manuscripts at http://www.hindawi.com
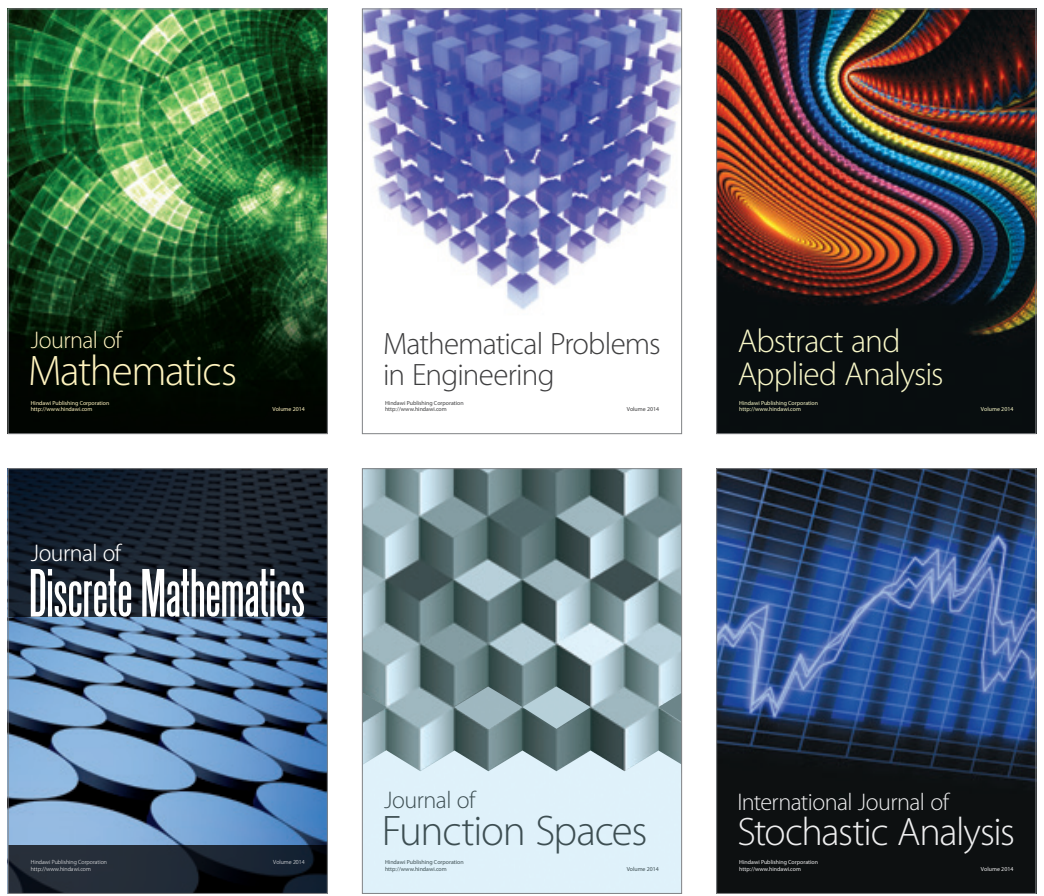

Journal of

Function Spaces

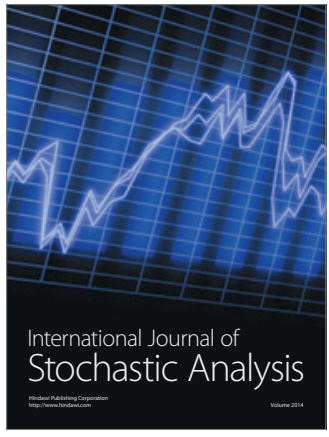

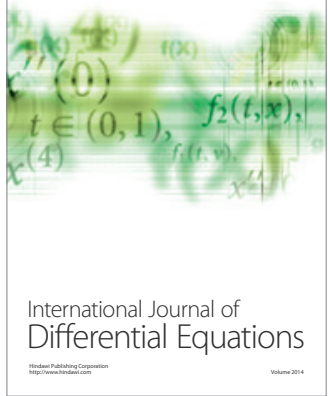
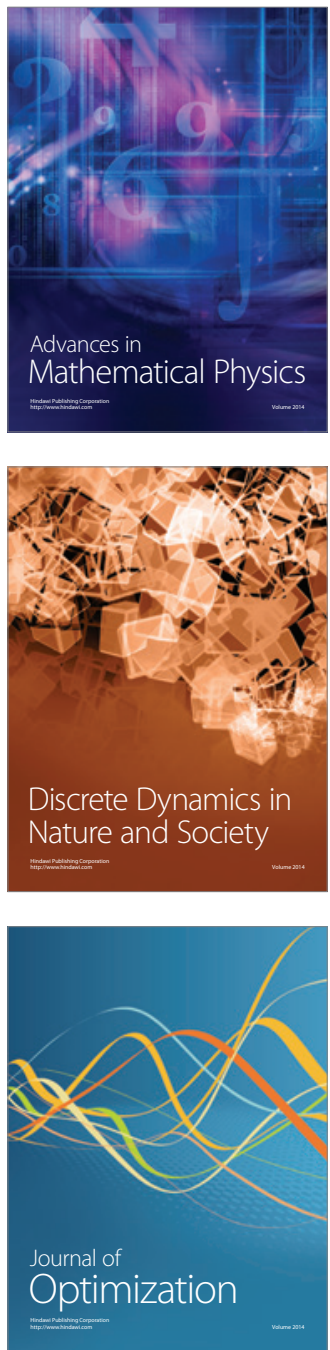